JOURNAL OF

FUNCTION SPACES AND APPLICATIONS

Volume 5, Number 3 (2007), 243-268
(C) 2007, Scientific Horizon http://www.jfsa.net

\title{
Trace theorems for Sobolev-Slobodeckij spaces with or without weights
}

\author{
Doyoon Kim
}

(Communicated by Hans Triebel)

2000 Mathematics Subject Classification. 46E35.

Keywords and phrases. weighted Sobolev spaces, Slobodeckij spaces, traces, extensions.

Abstract. We prove that the well-known trace theorem for weighted Sobolev spaces holds true under minimal regularity assumptions on the domain. Using this result, we prove the existence of a bounded linear right inverse of the trace operator for Sobolev-Slobodeckij spaces $W_{p}^{s}(\Omega)$ when $s-1 / p$ is an integer.

\section{Introduction}

The aim of this paper is to prove trace theorems for weighted Sobolev spaces $W_{p, r}^{k}(\Omega)$ and Sobolev-Slobodeckij spaces $W_{p}^{s}(\Omega)$ (without weights) under the weakest boundary regularity conditions - the minimal regularity assumptions on the boundary under which functions on the boundary are well defined. The weighted Sobolev spaces we consider in this paper are of the form (see [13])

$$
W_{p, r}^{k}(\Omega)=\left\{u: u, \rho(x)^{r / p} D^{\alpha} u \in L_{p}(\Omega) \quad \forall \alpha:|\alpha|=k\right\}
$$

where $r \in \mathbb{R}, k=1,2, \cdots$, and $\rho(x)$ is the distance from $x$ to the boundary of $\Omega$. 
Trace (on the boundary) and extension theorems for weighted Sobolev spaces or Sobolev-Slobodeckij spaces are well established if the domain is smooth enough. For example, a theorem in [13] states the following: If the boundary is $C^{l+1, \varepsilon}$, where $\varepsilon \in(0,1)$ and $l$ is an integer such that $k-(r+1) / p \leq l<k-(r+1) / p+1$, then the trace operator

$T: W_{p, r}^{k}(\Omega) \rightarrow \prod_{j=0}^{l-1} B_{p, p}^{k-\frac{r+1}{p}-j}(\partial \Omega), \quad T(u)=\left\{\left.u\right|_{\partial \Omega},\left.\frac{\partial u}{\partial N}\right|_{\partial \Omega}, \cdots,\left.\frac{\partial^{l-1} u}{\partial N^{l-1}}\right|_{\partial \Omega}\right\}$

is bounded and has a bounded right inverse, where $-1<r<k p-1$ and $N$ is the inner (or outer) normal vector field defined on $\partial \Omega$. For Sobolev-Slobodeckij spaces $W_{p}^{s}(\Omega)$, the results are almost complete because Marschall proved in [11] the following: If $\Omega$ is a Lipschitz domain and, in addition, a $C^{m, \delta}$-domain in case $s-1 / p \geq 1$, where $m$ is an integer, $0 \leq \delta<1$, and $m+\delta>s-1 / p$, then the trace operator

$$
T: W_{p}^{s}(\Omega) \rightarrow \prod_{j=0}^{l-1} B_{p, p}^{s-1 / p-j}(\partial \Omega)
$$

is bounded and, if $s-1 / p$ is not an integer, has a bounded right inverse, where $s>l-1+1 / p>0$. However, if $s-1 / p$ is an integer, it is assumed that the boundary is $C^{m, \delta}$, where $m+\delta>s$. See also [20] where one can find a remark that if $s-1 / p$ is an integer and the boundary does not preserve Markov's inequality, then it is unknown whether $T$ has a bounded linear right inverse.

In this paper, we show that both theorems in the above hold true under minimal regularity assumptions on the domain. More specifically, we prove that whenever the boundary is $C^{m, \delta}$, where $m+\delta>k-(r+1) / p$, the trace theorem for weighted Sobolev spaces holds true. This is done by constructing somewhat special diffeomorphisms which locally flatten the boundary. Then we prove that there is a bounded linear right inverse of the trace operator for Sobolev-Slobodeckij spaces even if $s-1 / p$ is an integer by using the trace theorem for weighted Sobolev spaces and applying an embedding theorem of weighted Sobolev spaces into Besov spaces.

Let us mention a few among many references for trace theorems for Sobolev-Slobodeckij spaces with or without weights. For more results and references for various spaces with or without weights, we refer to [17]. Trace theorems for weighted Sobolev spaces on a half space was dealt with in [10] and [19]. One can find trace theorems for weighted spaces on a bounded domain in [12], [16], and [13]. Also see [14] and [15]. For trace theorems for 
Sobolev-Slobodeckij spaces on a Lipschitz domain, in addition to [11], see [7] and [4].

This paper consists of two sections excluding this introduction. Trace theorems for Sobolev-Slobodeckij spaces with and without weights are proved in section 2 and 3 respectively. Throughout the paper, we use the following notations.

$\mathbb{R}^{d}$ is a $d$-dimensional Euclidean space and $\left(x_{1}, x^{\prime}\right)=\left(x_{1}, x_{2}, \cdots, x_{d}\right) \in$ $\mathbb{R}^{d}$. For a multi-index $\alpha=\left(\alpha_{1}, \cdots, \alpha_{d}\right)$,

$$
|\alpha|=\alpha_{1}+\cdots+\alpha_{d}, \quad D^{\alpha}=\frac{\partial^{|\alpha|}}{\partial x_{1}^{\alpha_{1}} \cdots \partial x_{d}^{\alpha_{d}}} .
$$

Various constants are denoted by $c$, their values may change from one occurrence to another.

\section{Traces of weighted Sobolev spaces}

We first introduce the definition of the weighted Sobolev spaces we are considering. As usual, $\Omega$ is a domain (open subset) in $\mathbb{R}^{d}$ and $\mathcal{D}^{\prime}(\Omega)$ is the set of all distributions on $\Omega$. We set $\rho(x)\left(=\rho_{\Omega}(x)\right)$ to be the distance function from $x \in \Omega$ to the boundary of $\Omega$.

Definition 2.1. Let $r \in \mathbb{R}, k=1,2, \cdots$, and $1<p<\infty$.

$$
W_{p, r}^{k}(\Omega)=\left\{u \in \mathcal{D}^{\prime}(\Omega):\|u\|_{L_{p}(\Omega)}+\sum_{|\alpha|=k}\left\|D^{\alpha} u\right\|_{L_{p, r}(\Omega)}<\infty\right\},
$$

where

$$
\|v\|_{L_{p, r}(\Omega)}=\left(\int_{\Omega}|v|^{p} \rho(x)^{r} d x\right)^{1 / p} .
$$

We also make use of the following weighted Sobolev spaces.

Definition 2.2. Let $k=1,2, \cdots$ and $1<p<\infty$.

$$
\mathcal{W}_{p, r}^{k}(\Omega)=\left\{u \in \mathcal{D}^{\prime}(\Omega): \sum_{0 \leq|\alpha| \leq k}\left\|D^{\alpha} u\right\|_{L_{p, r}(\Omega)}<\infty\right\} .
$$

The regularity of the boundary of a bounded domain $\Omega$ is described as follows.

Definition 2.3. Let $\kappa$ be a nonnegative integer and $\delta$ be a real number such that $0 \leq \delta<1$. For a bounded domain $\Omega$, we say $\partial \Omega \in C^{\kappa, \delta}$ if the 
following is satisfied: For each $x_{0} \in \partial \Omega$, there exist $r_{0}>0$ and a $C^{\kappa, \delta}$ function $h: \mathbb{R}^{d-1} \rightarrow \mathbb{R}$ such that - upon relabeling and re-orienting the coordinate axes if necessary - we have

$$
\Omega \cap B\left(x_{0}, r_{0}\right)=\left\{x \in B\left(x_{0}, r_{0}\right): x_{1}>h\left(x_{2}, \cdots, x_{d}\right)\right\} .
$$

When the above function $h$ is Lipschitz, we say $\partial \Omega \in C^{0,1}$. Without loss of generality, we always assume that $h$ has compact support.

If the boundary of a domain is regular enough (a Lipschitz domain is enough) and $r$ is in an appropriate range, then $\mathcal{W}_{p, r}^{k}(\Omega)$ (Definition 2.2) is not different from $W_{p, r}^{k}(\Omega)$ (Definition 2.1). This is stated in the following along with an embedding result.

Lemma 2.4. Let $-1<r \leq k p$ and $\partial \Omega \in C^{0,1}$. Then

(i) $W_{p, r}^{k}(\Omega)=\mathcal{W}_{p, r}^{k}(\Omega)$.

(ii) $W_{p, r}^{k}(\Omega) \hookrightarrow W_{p, r-j p}^{k-j}(\Omega) \hookrightarrow W_{p, r_{j}}^{k-j}(\Omega)$ for $0 \leq j \leq k$ and $r_{j} \geq$ $r-j p>-1$, where $\hookrightarrow$ means a continuous embedding.

The statements in the lemma may be found in the survey paper [13] (no proof, no boundary regularity is specified there) and references therein. In fact, they can be proved by using an argument based on Hardy's inequality (Theorem 5.1 in [9]). Also see [2], where similar results are proved using Hardy's inequality. In addition, one can use the results of [2] in proving the above lemma. Moreover, because of the first statement, the second statement follows from the properties of $\mathcal{W}_{p, r}^{k}(\Omega)$, which are well described in $[9]$.

Remark 2.5. If $\Omega$ is an unbounded domain, then $W_{p, r}^{k}(\Omega)$ is not necessarily the same space as $\mathcal{W}_{p, r}^{k}(\Omega)$ unless $r=0$.

To investigate traces (restriction) of functions to the boundary, we need function spaces defined on the boundary (see [11] or [17]).

Definition 2.6. Let $0 \leq s<1$ if $\partial \Omega \in C^{0,1}$ and $s<\kappa+\delta$ if $\partial \Omega \in C^{\kappa, \delta}$, where $\kappa$ is a positive integer and $0 \leq \delta<1$. By $B_{p, p}^{s}(\partial \Omega)$ we mean the collection of all functions $f$ defined on $\partial \Omega$ satisfying

$$
\left(\varphi^{\tau} f\right)\left(\Phi^{\tau}\left(0, y^{\prime}\right)\right) \in B_{p, p}^{s}\left(\mathbb{R}^{d-1}\right), \quad \tau=1, \cdots, M,
$$

where $\varphi^{\tau}, \tau=1, \cdots, M$, consist of a partition of unity and $\Phi^{\tau}$, $\tau=1, \cdots, M$, are local diffeomorphisms $\left(\Phi^{\tau}\left(0, y^{\prime}\right)\right.$ are Lipschitz or $C^{\kappa, \delta}$ functions depending on $s$ ). We set

$$
\|f\|_{B_{p, p}^{s}(\partial \Omega)}=\sum_{\tau=1}^{M}\left\|\left(\varphi^{\tau} f\right)\left(\Phi^{\tau}(0, \cdot)\right)\right\|_{B_{p, p}^{s}\left(\mathbb{R}^{d-1}\right)} .
$$


Remark 2.7. In the sense of equivalent norms, $B_{p, p}^{s}(\partial \Omega)$ is independent of the choice of a partition of unity and local diffeomorphisms. For more details about a partition of unity, local diffeomorphisms, and function spaces on the boundary, we refer to [17], where one can find the definition of $B_{p, p}^{s}\left(\mathbb{R}^{d-1}\right)$ as well. Also see the argument before the proof of Theorem 2.10 in this paper.

We investigate the trace and inverse extension theorem for weighted Sobolev spaces $W_{p, r}^{k}(\Omega), k=1,2, \cdots, 1<p<\infty$, under the following assumptions.

\section{Assumption 2.8 .}

1. The real number $r$ satisfies $-1<r<k p-1$. Thus one can find a positive integer $l$ satisfying

$$
k-\frac{r+1}{p} \leq l<k-\frac{r+1}{p}+1 .
$$

2. The domain $\Omega$ is bounded and the boundary of $\Omega$ satisfies one of the following conditions.

(i) $\partial \Omega \in C^{0,1}$ if $k-\frac{r+1}{p}<1$, i.e., $l=1$.

(ii) $\partial \Omega \in C^{l, \delta}$ if $k-\frac{r+1}{p}=l$, where $\delta$ is a real number such that $0<\delta<1$.

(iii) $\partial \Omega \in C^{l-1, \delta}$ if $1<k-\frac{r+1}{p}<l<k-\frac{r+1}{p}+1$, where $\delta$ is a real number such that $0<\delta<1$ and $k-\frac{r+1}{p}<l-1+\delta$.

In particular, if $k-\frac{r+1}{p} \geq 1$, then we can say that $\partial \Omega \in C^{m, \delta}$, where $m$ is the integer $\left[k-\frac{r+1}{p}\right]$, which is the greatest integer less than or equal to $k-\frac{r+1}{p}$, and $\delta$ is a real number satisfying $0<\delta<1$ and

$$
k-\frac{r+1}{p}<m+\delta<k-\frac{r+1}{p}+1 .
$$

Let $W_{p, r}^{k}(\Omega)$ satisfy Assumption 2.8 and $N$ be the unit inner normal vector field defined on $\partial \Omega$. For a function $u \in W_{p, r}^{k}(\Omega) \cap C^{l-1}(\bar{\Omega})$, where $l$ is the integer in Assumption 2.8, define $T_{j}$ by

$$
T_{j} u(x)=\left.\sum_{|\alpha|=j}\left(D^{\alpha} u\right)\right|_{\partial \Omega}(x) N^{\alpha}(x) \quad j=0, \cdots, l-1,
$$

where $N^{\alpha}(x)=N_{1}^{\alpha_{1}}(x) \cdots N_{d}^{\alpha_{d}}(x)$. Then we set $T$, called the trace operator, to be

$$
T u=\left\{T_{0} u, \cdots, T_{l-1} u\right\} .
$$


Remark 2.9. To define $T_{1} u$, one may also use the notation $\partial u / \partial N$, which is

$$
\frac{\partial u}{\partial N}=\sum_{|\alpha|=1} D^{\alpha} u N^{\alpha} \quad \text { on } \quad \partial \Omega .
$$

In case $j \geq 2$, the notation $\partial^{j} u / \partial N^{j}$ may not be as clear as $\partial u / \partial N$. Indeed, let $N(x)$ be defined in a neighborhood of $\partial \Omega$ and set

$$
\frac{\partial^{j} u}{\partial N^{j}}=\frac{\partial}{\partial N}\left(\frac{\partial^{j-1} u}{\partial N^{j-1}}\right), \quad j \geq 2 .
$$

Then it is not necessarily true that

$$
\frac{\partial^{j} u}{\partial N^{j}}=\sum_{|\alpha|=j} D^{\alpha} u N^{\alpha} \quad \text { on } \quad \partial \Omega
$$

unless we have

$$
\frac{\partial N_{i}}{\partial N}=\cdots=\frac{\partial^{j-1} N_{i}}{\partial N^{j-1}}=0 \quad \text { on } \quad \partial \Omega \quad \text { for all } i \in\{1, \cdots, d\} .
$$

However, the above condition is satisfied by an appropriate extension of the normal vector $N(x)$ (see Lemma 2.17 below), so one may define $T_{j} u$ using the notation $\partial^{j} u / \partial N^{j}$ assuming that $N$ is properly extended.

The following theorem is the main result of this section.

Theorem 2.10. Under Assumption 2.8, the operator $T$ defined in (1) is a bounded operator from

$$
W_{p, r}^{k}(\Omega) \quad \text { onto } \quad \prod_{j=0}^{l-1} B_{p, p}^{k-\frac{r+1}{p}-j}(\partial \Omega)
$$

satisfying

$$
\sum_{j=0}^{l-1}\left\|T_{j} u\right\|_{B_{p, p}^{k-\frac{r+1}{p}-j}(\partial \Omega)} \leq c\|u\|_{W_{p, r}^{k}(\Omega)},
$$

where $c$ is independent of $u \in W_{p, r}^{k}(\Omega)$.

Moreover, for a given $\left\{g_{0}, \cdots, g_{l-1}\right\} \in \prod_{j=0}^{l-1} B_{p, p}^{k-\frac{r+1}{p}-j}(\partial \Omega)$, there exists a function $u \in W_{p, r}^{k}(\Omega)$ such that

$$
\|u\|_{W_{p, r}^{k}(\Omega)} \leq c \sum_{j=0}^{l-1}\left\|g_{j}\right\|_{B_{p, p}^{k-\frac{r+1}{p}-j}(\partial \Omega)}, \quad T u=\left\{g_{0}, \cdots, g_{l-1}\right\} .
$$


To prove this theorem, we need a series of observations. The first one is called Faà di Bruno's Formula, which is proved in full generality in [3]. We present here a simple version of the formula, which can be proved using induction.

Lemma 2.11. Let $v$ be a scalar valued function and $u(x)=v(\Psi(x))$, where $\Psi(x)=\left(\Psi_{1}(x), \cdots, \Psi_{d}(x)\right)$. Assume that $u$ and $\Psi$ are sufficiently smooth. Then for a multi-index $\alpha,|\alpha| \geq 1, D^{\alpha} u(x)$ consists of the sum of terms each of which is a constant times a function of the form

$$
\left(D^{\beta} v\right)(\Psi(x)) \prod_{j=1}^{|\alpha|+1-|\beta|} \zeta_{\beta, j}(x),
$$

where $\beta$ is a multi-index with $1 \leq|\beta| \leq|\alpha|$ and $\zeta_{\beta, j}(x), j=1, \cdots,|\alpha|+1-$ $|\beta|$, are defined as follows: For each multi-index $\beta$, there exist nonnegative integers $a_{\beta, j}$ (denoted by $a_{j}$ for simplicity), $j=1, \cdots,|\alpha|+1-|\beta|$, such that

$$
\sum_{j=1}^{|\alpha|+1-|\beta|} a_{j}=|\beta|, \quad \sum_{j=1}^{|\alpha|+1-|\beta|} j a_{j}=|\alpha|,
$$

and $\zeta_{\beta, j}(x)$ is of the form

$$
\zeta_{\beta, j}(x)=\left\{\begin{array}{cl}
1 & \text { if } a_{j}=0 \\
\prod_{\substack{k=1, \cdots, a_{j} \\
\left|\gamma_{k}\right|=j}} D^{\gamma_{k}} \Psi_{i(k)}(x) & \text { if } a_{j} \geq 1
\end{array},\right.
$$

where $\gamma_{k}, k=1, \cdots, a_{j}$, are multi-indices and $i(k) \in\{1, \cdots, d\}$.

We make use of the following lemma especially when the function $\varrho(x)$ is the distance function $\rho(x)=\operatorname{dist}(x, \partial \Omega)$.

Lemma 2.12. Let $\Omega$ be an open subset in $\mathbb{R}^{d}$ and $\varrho$ be a non-negative function defined on $\Omega$. Assume that $\Psi(x)=\left(\Psi_{1}(x), \cdots, \Psi_{d}(x)\right)$ is a sufficiently smooth function defined on $\Omega$ and $\zeta_{\beta, j}(x), j=1, \cdots,|\alpha|+$ $1-|\beta|$, are those defined in Lemma 2.11, where $\alpha$ and $\beta$ are multi-indices such that $|\alpha| \geq 1$ and $1 \leq|\beta| \leq|\alpha|$. Assume that we have

(i) $\varrho(x)^{|\gamma|-1} D^{\gamma} \Psi(x)$ is bounded in $\Omega$ for $1 \leq|\gamma| \leq|\alpha|$.

Then

$$
\varrho(x)^{|\alpha|-|\beta|} \prod_{j=1}^{|\alpha|+1-|\beta|} \zeta_{\beta, j}(x)
$$

is bounded in $\Omega$.

Let $m$ be a positive integer and $\delta$ be a real number such that $0 \leq \delta \leq 1$. Assume that we have the following two conditions as well as (i) in the above. 
(ii) $D^{\gamma} \Psi(x)$ is bounded in $\Omega$ for $1 \leq|\gamma| \leq m$.

(iii) $\varrho(x)^{|\gamma|-m-\delta} D^{\gamma} \Psi(x)$ is bounded in $\Omega$ for $m+1 \leq|\gamma| \leq|\alpha|$.

Then for $|\alpha| \geq m+1$ and $1 \leq|\beta| \leq|\alpha|-m$,

$$
\varrho(x)^{|\alpha|-\max \{|\beta|, m\}-\delta} \prod_{j=1}^{|\alpha|+1-|\beta|} \zeta_{\beta, j}(x)
$$

is bounded in $\Omega$.

Proof. Note that

$\varrho(x)^{|\alpha|-|\beta|} \prod_{j=1}^{|\alpha|+1-|\beta|} \zeta_{\beta, j}(x)=\varrho(x)^{|\alpha|-|\beta|} \prod_{j=1}^{|\alpha|+1-|\beta|} \varrho(x)^{(1-j) a_{j}} \varrho(x)^{(j-1) a_{j}} \zeta_{\beta, j}(x)$.

Also note that for $a_{j} \geq 1$,

$$
\varrho(x)^{(j-1) a_{j}} \zeta_{\beta, j}(x)=\prod_{\substack{k=1, \cdots, a_{j} \\\left|\gamma_{k}\right|=j}} \varrho(x)^{\left|\gamma_{k}\right|-1} D^{\gamma_{k}} \Psi_{i(k)}(x),
$$

which is bounded by the assumption. Thus we need only verify that

$$
\varrho(x)^{|\alpha|-|\beta|} \prod_{j=1}^{|\alpha|+1-|\beta|} \varrho(x)^{(1-j) a_{j}}
$$

is bounded, which is true because

$$
|\alpha|-|\beta|+\sum_{j=1}^{|\alpha|+1-|\beta|}(1-j) a_{j}=0 .
$$

Now we prove the second assertion. Notice that, for points where $\varrho(x) \geq 1$,

$$
\varrho(x)^{|\alpha|-\max \{|\beta|, m\}-\delta}\left|\prod_{j=1}^{|\alpha|+1-|\beta|} \zeta_{\beta, j}(x)\right| \leq \varrho(x)^{|\alpha|-|\beta|}\left|\prod_{j=1}^{|\alpha|+1-|\beta|} \zeta_{\beta, j}(x)\right|,
$$

and the right hand side of this equality is shown to be bounded in the above. For points where $\varrho(x)<1$, observe that $m+1 \leq|\alpha|+1-|\beta|$ and

$$
\varrho(x)^{|\alpha|-\max \{|\beta|, m\}-\delta} \prod_{j=1}^{|\alpha|+1-|\beta|} \zeta_{\beta, j}(x)
$$


$=\varrho(x)^{|\alpha|-\max \{|\beta|, m\}-\delta} \prod_{j=1}^{m} \zeta_{\beta, j}(x) \prod_{j=m+1}^{|\alpha|+1-|\beta|} \varrho(x)^{(m+\delta-j) a_{j}} \varrho(x)^{(j-m-\delta) a_{j}} \zeta_{\beta, j}(x)$.

By the assumption it follows that

$$
\prod_{j=1}^{m} \zeta_{\beta, j}(x) \prod_{j=m+1}^{|\alpha|+1-|\beta|} \varrho(x)^{(j-m-\delta) a_{j}} \zeta_{\beta, j}(x)
$$

is bounded. Therefore, we need only check that

$$
|\alpha|-\max \{|\beta|, m\}-\delta+\sum_{j=m+1}^{|\alpha|+1-|\beta|}(m+\delta-j) a_{j}
$$

is non-negative. If $\max \{|\beta|, m\}=m$, then (4) equals

$$
(m+\delta)\left(\sum_{j=m+1}^{|\alpha|+1-|\beta|} a_{j}-1\right)+\sum_{j=1}^{m} j a_{j}
$$

which is non-negative if one of $a_{m+1}, \cdots, a_{|\alpha|+1-|\beta|}$ is a positive integer. If $a_{m+1}=\cdots=a_{|\alpha|+1-|\beta|}=0$, it equals $|\alpha|-m-\delta$ because $|\alpha|=$ $\sum_{j=1}^{|\alpha|+1-|\beta|} j a_{j}=\sum_{j=1}^{m} j a_{j}$. This is non-negative since $|\alpha| \geq m+1$. If $\max \{|\beta|, m\}=|\beta|$, then (4) equals

$$
-\delta+\sum_{j=1}^{m}(j-1) a_{j}+(m+\delta-1) \sum_{j=m+1}^{|\alpha|+1-|\beta|} a_{j},
$$

which is non-negative if one of $a_{m+1}, \cdots, a_{|\alpha|+1-|\beta|}$ is a positive integer. If $a_{m+1}=\cdots=a_{|\alpha|+1-|\beta|}=0$, it is equal to $|\alpha|-|\beta|-\delta$. This is non-negative since $|\beta| \leq|\alpha|-m$ and $m \geq 1$. The lemma is proved.

Remark 2.13. We see from the proof that, in case $|\beta| \geq 2$, the above lemma still holds if the assumptions (i) and (iii) are replaced with

(i') $\varrho(x)^{|\gamma|-1} D^{\gamma} \Psi(x)$ is bounded in $\Omega$ for $1 \leq|\gamma| \leq|\alpha|-1$.

(iii') $\varrho(x)^{|\gamma|-m-\delta} D^{\gamma} \Psi(x)$ is bounded in $\Omega$ for $m+1 \leq|\gamma| \leq|\alpha|-1$.

The proof of Theorem 2.10 relies on somewhat special diffeomorphisms which locally flatten the boundary of a given domain. To construct such diffeomorphisms we follow ideas in $[5,8]$. Let $h$ be a function with compact support such that $h \in C^{m, \delta}\left(\mathbb{R}^{d-1}\right)$, where $\delta=1$ if $m=0$, or $0 \leq \delta<1$ if $m$ is a positive integer. We define a function $H$ in $\mathbb{R}^{d}$ to be 


$$
H\left(y_{1}, y^{\prime}\right)=\int_{\mathbb{R}^{d-1}} h\left(y^{\prime}-y_{1} z^{\prime}\right) \phi\left(z^{\prime}\right) d z^{\prime},
$$

where $\phi \in C_{0}^{\infty}\left(\mathbb{R}^{d-1}\right)$ satisfies $\int_{\mathbb{R}^{d-1}} \phi\left(z^{\prime}\right) d z^{\prime}=1$.

First assume that $h \in C^{0,1}\left(\mathbb{R}^{d-1}\right)$. It is clear that $H \in C^{0,1}\left(\mathbb{R}^{d}\right)$. Let $\alpha$ be a multi-index such that $|\alpha| \geq 1$. Then for $y_{1} \neq 0$, we have

$$
\begin{gathered}
D^{\alpha} H\left(y_{1}, y^{\prime}\right)=D^{\alpha}\left[y_{1}^{1-d} \int_{\mathbb{R}^{d-1}} h\left(z^{\prime}\right) \phi\left(\left(y^{\prime}-z^{\prime}\right) / y_{1}\right) d z^{\prime}\right] \\
=y_{1}^{-|\alpha|} \int_{\mathbb{R}^{d-1}} h\left(y^{\prime}-y_{1} z^{\prime}\right) \phi_{\alpha}\left(z^{\prime}\right) d z^{\prime},
\end{gathered}
$$

where $\phi_{\alpha}\left(z^{\prime}\right) \in C_{0}^{\infty}\left(\mathbb{R}^{d-1}\right)$ and $\int_{\mathbb{R}^{d-1}} \phi_{\alpha}\left(z^{\prime}\right) d z^{\prime}=0$. Moreover,

$$
\left|D^{\alpha} H\left(y_{1}, y^{\prime}\right)\right|=\left|y_{1}^{-|\alpha|} \int_{\mathbb{R}^{d-1}}\left[h\left(y^{\prime}-y_{1} z^{\prime}\right)-h\left(y^{\prime}\right)\right] \phi_{\alpha}\left(z^{\prime}\right) d z^{\prime}\right| \leq c\left|y_{1}\right|^{-|\alpha|+1} .
$$

Now assume that $h \in C^{m, \delta}\left(\mathbb{R}^{d-1}\right)$, where $m=1,2, \cdots, 0 \leq \delta<1$. Let $\beta$ be a multi-index such that $1 \leq|\beta| \leq m$ and

$$
\beta=\left(\beta_{1}, \cdots, \beta_{d}\right)=\left(\beta_{1}, 0, \cdots, 0\right)+\left(0, \beta_{2}, \cdots, \beta_{d}\right)=:\left(\beta_{1}, 0, \cdots, 0\right)+\beta^{\prime} .
$$

Then

$$
\begin{aligned}
D^{\beta} H\left(y_{1}, y^{\prime}\right) & =\frac{\partial^{\beta_{1}}}{\partial y_{1}^{\beta_{1}}} D^{\beta^{\prime}} H\left(y_{1}, y^{\prime}\right)=\frac{\partial^{\beta_{1}}}{\partial y_{1}^{\beta_{1}}} \int_{\mathbb{R}^{d-1}} D^{\beta^{\prime}} h\left(y^{\prime}-y_{1} z^{\prime}\right) \phi\left(z^{\prime}\right) d z^{\prime} \\
& =\sum_{|\gamma|=\beta_{1}} \int_{\mathbb{R}^{d-1}}\left(D^{\beta^{\prime}+\gamma} h\right)\left(y^{\prime}-y_{1} z^{\prime}\right)\left(-z^{\prime}\right)^{\gamma} \phi\left(z^{\prime}\right) d z^{\prime},
\end{aligned}
$$

where $\gamma$ is a $(d-1)$-dimensional multi-index and $\left(-z^{\prime}\right)^{\gamma}=\left(-z_{2}\right)^{\gamma_{2}} \cdots\left(-z_{d}\right)^{\gamma_{d}}$. From this calculation it follows that $H\left(y_{1}, y^{\prime}\right) \in C^{m, \delta}\left(\mathbb{R}^{d}\right)$. Set $\phi_{\gamma}\left(z^{\prime}\right):=$ $\left(-z^{\prime}\right)^{\gamma} \phi\left(z^{\prime}\right)$, and let $\beta$ and $\alpha$ be multi-indices such that $\beta=\left(\beta_{1}, 0, \cdots, 0\right)+$ $\beta^{\prime},|\beta|=m$, and $|\alpha| \geq 1$. Then for $y_{1} \neq 0$, we have

$$
\begin{gathered}
D^{\alpha+\beta} H\left(y_{1}, y^{\prime}\right)=D^{\alpha}\left[\sum_{|\gamma|=\beta_{1}} \int_{\mathbb{R}^{d-1}}\left(D^{\beta^{\prime}+\gamma} h\right)\left(y^{\prime}-y_{1} z^{\prime}\right) \phi_{\gamma}\left(z^{\prime}\right) d z^{\prime}\right] \\
=D^{\alpha}\left[y_{1}^{1-d} \sum_{|\gamma|=\beta_{1}} \int_{\mathbb{R}^{d-1}}\left(D^{\beta^{\prime}+\gamma} h\right)\left(z^{\prime}\right) \phi_{\gamma}\left(\left(y^{\prime}-z^{\prime}\right) / y_{1}\right) d z^{\prime}\right] \\
=y_{1}^{-|\alpha|} \sum_{|\gamma|=\beta_{1}} \int_{\mathbb{R}^{d-1}}\left(D^{\beta^{\prime}+\gamma} h\right)\left(y^{\prime}-y_{1} z^{\prime}\right) \phi_{\alpha, \gamma}\left(z^{\prime}\right) d z^{\prime}
\end{gathered}
$$


where $\phi_{\alpha, \gamma}\left(z^{\prime}\right) \in C_{0}^{\infty}\left(\mathbb{R}^{d-1}\right)$ and $\int_{\mathbb{R}^{d-1}} \phi_{\alpha, \gamma}\left(z^{\prime}\right) d z^{\prime}=0$. Moreover,

$$
\begin{gathered}
\left|D^{\alpha+\beta} H\left(y_{1}, y^{\prime}\right)\right| \\
=\left|y_{1}^{-|\alpha|} \sum_{|\gamma|=\beta_{1}} \int_{\mathbb{R}^{d-1}}\left[\left(D^{\beta^{\prime}+\gamma} h\right)\left(y^{\prime}-y_{1} z^{\prime}\right)-\left(D^{\beta^{\prime}+\gamma} h\right)\left(y^{\prime}\right)\right] \phi_{\alpha, \gamma}\left(z^{\prime}\right) d z^{\prime}\right| \\
\leq c\left|y_{1}\right|^{-|\alpha|+\delta} .
\end{gathered}
$$

By the properties of $H$ shown in the above, we can choose an appropriate $\varepsilon>0$ such that

$$
\left|\frac{\partial}{\partial y_{1}}\left[H\left(\varepsilon y_{1}, y^{\prime}\right)\right]\right|<1 / 2
$$

for all points $\left(y_{1}, y^{\prime}\right), y_{1} \neq 0$, in $\mathbb{R}^{d}$ (only in case $m=0$ and $\delta=1$, $\partial H / \partial y_{1}$ may not be defined on some points in $\left.\left\{\left(0, y^{\prime}\right): y^{\prime} \in \mathbb{R}^{d-1}\right\}\right)$. With this $\varepsilon$, we define

$$
\Phi(y)=\left(y_{1}+H\left(\varepsilon y_{1}, y^{\prime}\right), y^{\prime}\right)
$$

Note that

$$
\frac{\partial \Phi_{1}}{\partial y_{1}}=1+\varepsilon \frac{\partial H}{\partial y_{1}}\left(\varepsilon y_{1}, y^{\prime}\right)>1 / 2
$$

for all points $\left(y_{1}, y^{\prime}\right), y_{1} \neq 0$, in $\mathbb{R}^{d}$. This and the fact that $H \in C^{m, \delta}\left(\mathbb{R}^{d}\right)$ indicate that $y_{1}+H\left(\varepsilon y_{1}, y^{\prime}\right)$ is an increasing function of $y_{1}$. From this and the definition of $\Phi$, we see that $\Phi$ is one-to-one from $\mathbb{R}^{d}$ onto itself. Therefore, there exists a function $\Psi$ defined in $\mathbb{R}^{d}$ such that $\Psi=\Phi^{-1}$.

Lemma 2.14. Let $h$ be a function with compact support defined in $\mathbb{R}^{d-1}$ such that $h \in C^{m, \delta}\left(\mathbb{R}^{d-1}\right)$, where $\delta=1$ if $m=0$, or $0 \leq \delta<1$ if $m=1,2, \cdots$. Let $U=\left\{\left(x_{1}, x^{\prime}\right) \in \mathbb{R}^{d}: x_{1}>h\left(x^{\prime}\right)\right\}$ and $\rho(x)=\operatorname{dist}(x, \partial U)$. Then the functions $\Psi(x)$ and $\Phi(y)$ defined in the above satisfy

(i) $\Psi \in C_{l o c}^{m, \delta}\left(\mathbb{R}^{d}\right)$.

(ii) For $|\alpha| \geq 1$,

$$
\sup _{x \in U} \rho(x)^{|\alpha|-1}\left|D^{\alpha} \Psi(x)\right|<\infty .
$$

(iii) For $|\alpha| \geq 1$ and $|\beta|=m$,

$$
\sup _{x \in U} \rho(x)^{|\alpha|-\delta}\left|D^{\alpha+\beta} \Psi(x)\right|<\infty .
$$

(i') $\Phi \in C_{l o c}^{m, \delta}\left(\mathbb{R}^{d}\right)$. 
(ii') For $|\alpha| \geq 1$,

$$
\sup _{y \in \mathbb{R}_{+}^{d}} y_{1}^{|\alpha|-1}\left|D^{\alpha} \Phi(y)\right|<\infty .
$$

(iii' ${ }^{\prime}$ For $|\alpha| \geq 1$ and $|\beta|=m$,

$$
\sup _{y \in \mathbb{R}_{+}^{d}} y_{1}^{|\alpha|-\delta}\left|D^{\alpha+\beta} \Phi(y)\right|<\infty .
$$

Proof. The statements $\left(\mathrm{i}^{\prime}\right),\left(\mathrm{ii}^{\prime}\right)$, and (iii') follow directly from the calculations shown above. We prove that $\Psi$ satisfies (i), (ii), and (iii). First note that

$$
\Psi_{i}(x)=x_{i} \quad \text { for } \quad i=2, \cdots, d,
$$

thus we concentrate on $D^{\alpha} \Psi_{1}(x)$. Using the fact that $\Psi=\Phi^{-1}$ and (6), we have

$$
\Psi_{1}(x)=x_{1}-H\left(\varepsilon \Psi_{1}(x), x^{\prime}\right) .
$$

Considering $F\left(x, y_{1}\right)=x_{1}-y_{1}-H\left(\varepsilon y_{1}, x^{\prime}\right)$ and the implicit function theorem we can justify that $\Psi_{1}(x)$ is infinitely differentiable at any point apart from $\partial U$. In addition, if $m \geq 1$, then $\Psi_{1}(x)$ is $m$ times continuously differentiable in $\mathbb{R}^{d}$. Set $\tilde{H}\left(y_{1}, y^{\prime}\right)=H\left(\varepsilon y_{1}, y^{\prime}\right)$. By differentiating both sides of (7) we have

$$
\begin{aligned}
& \frac{\partial \Psi_{1}}{\partial x_{1}}(x)=1-\frac{\partial \tilde{H}}{\partial y_{1}}(\Psi(x)) \frac{\partial \Psi_{1}}{\partial x_{1}}(x), \\
& \frac{\partial \Psi_{1}}{\partial x_{i}}(x)=-\frac{\partial \tilde{H}}{\partial y_{1}}(\Psi(x)) \frac{\partial \Psi_{1}}{\partial x_{i}}(x)-\frac{\partial \tilde{H}}{\partial y_{i}}(\Psi(x)), \quad i=2, \cdots, d .
\end{aligned}
$$

For $|\alpha| \geq 2$, we have

$$
D^{\alpha} \Psi_{1}(x)=-D^{\alpha}[\tilde{H}(\Psi(x))] .
$$

By Lemma 2.11 the right hand side in the above is the sum of terms each of which is a constant times a term of the form

$$
\left(D^{\beta} \tilde{H}\right)(\Psi(x)) \prod_{j=1}^{|\alpha|+1-|\beta|} \zeta_{\beta, j}(x),
$$

where $\beta$ is a multi-index with $1 \leq|\beta| \leq|\alpha|$ and $\zeta_{\beta, j}(x), j=1, \cdots,|\alpha|+$ $1-|\beta|$, are those in Lemma 2.11. Especially, if $|\beta|=1$, the only term we 
have is

$$
\frac{\partial \tilde{H}}{\partial y_{1}}(\Psi(x)) D^{\alpha} \Psi_{1}(x)
$$

Therefore, for $|\alpha| \geq 2$

$$
D^{\alpha} \Psi_{1}(x)=\left(1+\frac{\partial \tilde{H}}{\partial y_{1}}(\Psi(x))\right)^{-1} \sum_{2 \leq|\beta| \leq|\alpha|} c_{\beta}\left(D^{\beta} \tilde{H}\right)(\Psi(x)) \prod_{j=1}^{|\alpha|+1-|\beta|} \zeta_{\beta, j}(x),
$$

where $c_{\beta}$ are appropriate constants. Observe that, in case $2 \leq|\beta| \leq|\alpha|$, the orders of derivatives of $\Psi(x)$ which appears in (9) are less than or equal to $|\alpha|-1$.

If $m=0$ and $\delta=1$, then (8) and (5) imply that $\partial \Psi_{1} / \partial x_{i}, i=1, \cdots, d$, are bounded in $\mathbb{R}^{d} \backslash \partial U$. Then it follows that $\Psi_{1}$ is Lipschitz on $U$ and $\mathbb{R}^{d} \backslash \bar{U}$. Considering the fact that $\Psi=\Phi^{-1}$, we see that $\Psi$ is Lipschitz on $\mathbb{R}^{d}$.

If $m \geq 1$ and $0 \leq \delta<1$, then using (5), (8), (10), and the fact that $H \in C^{m, \delta}\left(\mathbb{R}^{d}\right)$, we prove that $\Psi \in C_{\text {loc }}^{m, \delta}\left(\mathbb{R}^{d}\right)$ (see Theorem A.9 in [6]). Therefore, (i) is proved. Moreover, for $1 \leq|\alpha| \leq m$,

$$
\sup _{x \in \mathbb{R}^{d}}\left|D^{\alpha} \Psi(x)\right|<\infty .
$$

To prove (ii) and (iii) we first see that, for any $x \in U$, there exist positive constants $c_{1}$ and $c_{2}$ such that

$$
c_{1} y_{1} \leq \rho(x) \leq c_{2} y_{1},
$$

where $y_{1}$ is the first coordinate of $\Psi(x)$.

Indeed, for $x=\left(x_{1}, x^{\prime}\right) \in U$, if we let $\left(y_{1}, y^{\prime}\right)=\Psi(x)$, then since $\Phi$ is Lipschitz on $\mathbb{R}^{d}$,

$$
\rho(x) \leq\left|\left(x_{1}, x^{\prime}\right)-\left(h\left(x^{\prime}\right), x^{\prime}\right)\right|=\left|\Phi\left(y_{1}, y^{\prime}\right)-\Phi\left(0, y^{\prime}\right)\right| \leq c y_{1} .
$$

Thus the second inequality in (12) holds true. For the proof of the first inequality in (12), note that if $x \in U$, then

$$
\rho(x)=\inf \{|x-z|: z \in \partial U\} .
$$

Thus, for any $\varepsilon^{\prime}>0$, there exists $\bar{z} \in \partial U$ such that $|x-\bar{z}|<\rho(x)+\varepsilon^{\prime}$. Let $y=\left(y_{1}, y^{\prime}\right)=\Psi(x)$ and $\bar{y}=\left(0, \bar{y}^{\prime}\right)=\Psi(\bar{z})$, then

$$
c|x-\bar{z}| \geq|\Psi(x)-\Psi(\bar{z})|=\left|\left(y_{1}, y^{\prime}\right)-\left(0, \bar{z}^{\prime}\right)\right| \geq y_{1}
$$

This proves the first inequality in (12). 
We now prove that $\Psi$ satisfies (ii). It is clear that (ii) holds true for $|\alpha|=1$ since $\partial \Psi(x) / \partial x_{i}, i=1, \cdots, d$, are bounded in $U$. To proceed using induction, we assume that (ii) holds for multi-indices of degree $\leq k$. Set $\alpha$ to be a multi-index of order $k+1$. From (10) each term of

$$
\rho(x)^{|\alpha|-1} D^{\alpha} \Psi_{1}(x)\left(1+\frac{\partial \tilde{H}}{\partial y_{1}}(\Psi(x))\right)
$$

is a constant times a term of the form

$$
\rho(x)^{|\alpha|-1}\left(D^{\beta} \tilde{H}\right)(\Psi(x)) \prod_{j=1}^{|\alpha|+1-|\beta|} \zeta_{\beta, j}(x),
$$

where $2 \leq|\beta| \leq|\alpha|=k+1$. This is equal to

$$
\rho(x)^{|\beta|-1}\left(D^{\beta} \tilde{H}\right)(\Psi(x)) \rho(x)^{|\alpha|-|\beta|} \prod_{j=1}^{|\alpha|+1-|\beta|} \zeta_{\beta, j}(x) .
$$

Since the orders of derivatives of $\Psi$ in $\zeta_{\beta, j}$ are less than or equal to $|\alpha|-1$, by applying Lemma 2.12 along with the induction hypothesis and Remark 2.13 , we have that

$$
\rho(x)^{|\alpha|-|\beta|} \prod_{j=1}^{|\alpha|+1-|\beta|} \zeta_{\beta, j}(x)
$$

is bounded. From the properties of $H$ and (12), we know that $\rho(x)^{|\beta|-1}\left(D^{\beta} \tilde{H}\right)(\Psi(x))$ is bounded. Finally, upon recalling (5) we see that $\rho(x)^{|\alpha|-1}(x) D^{\alpha} \Psi_{1}(x)$ is bounded if $|\alpha|=k+1$. This finishes the proof of (ii).

Let us prove (iii). If $m=0$ and $\delta=1$, then (iii) is identical to (ii). Thus we consider only the case $m \geq 1$ and $0 \leq \delta<1$. To make use of induction again, we first prove that (iii) is true when $|\alpha|=1$. Set $\alpha^{\prime}=\alpha+\beta$, where $|\alpha|=1$ and $|\beta|=m$. As above, we need that

$$
\rho(x)^{1-\delta}\left(D^{\beta^{\prime}} \tilde{H}\right)(\Psi(x)) \prod_{j=1}^{\left|\alpha^{\prime}\right|+1-\left|\beta^{\prime}\right|} \zeta_{\beta^{\prime}, j}(x), \quad 2 \leq\left|\beta^{\prime}\right| \leq\left|\alpha^{\prime}\right|,
$$

is bounded. Since $\left|\beta^{\prime}\right| \geq 2$, we have $\left|\alpha^{\prime}\right|+1-\left|\beta^{\prime}\right| \leq m$. This means that the orders of derivatives of $\Psi$ in $\zeta_{\beta^{\prime}, j}$ are less than or equal to $m$, thus by (11)

$$
\prod_{j=1}^{\left|\alpha^{\prime}\right|+1-\left|\beta^{\prime}\right|} \zeta_{\beta^{\prime}, j}(x)
$$


is bounded. To see that $\rho(x)^{1-\delta}\left(D^{\beta^{\prime}} \tilde{H}\right)(\Psi(x))$ is bounded, we consider two cases. If $\rho(x) \geq 1$, we have

$$
\rho(x)^{1-\delta}\left|\left(D^{\beta^{\prime}} \tilde{H}\right)(\Psi(x))\right| \leq \rho(x)^{\left|\beta^{\prime}\right|-1}\left|\left(D^{\beta^{\prime}} \tilde{H}\right)(\Psi(x))\right|
$$

(recall that $\left|\beta^{\prime}\right| \geq 2$ ). By the properties of $H$ and (12), the left hand side is bounded. For points where $\rho(x) \leq 1$, we know that $\left(D^{\beta^{\prime}} \tilde{H}\right)(\Psi(x))$ is bounded if $2 \leq\left|\beta^{\prime}\right| \leq m$ and $\rho(x)^{1-\delta}\left(D^{\beta^{\prime}} \tilde{H}\right)(\Psi(x))$ is bounded if $\left|\beta^{\prime}\right|=m+1$. Hence we have proved that (13) is bounded, so the assertion (iii) holds true when $|\alpha|=1$. Now we assume that (iii) are satisfied when $1 \leq|\alpha| \leq k$. Let $\alpha$ and $\beta$ be multi-indices such that $|\alpha|=k+1$ and $|\beta|=m$. Set $\alpha^{\prime}=\alpha+\beta$. Then what we need is

$$
\sup _{x \in U} \rho(x)^{\left|\alpha^{\prime}\right|-m-\delta}\left|D^{\alpha^{\prime}} \Psi_{1}(x)\right|<\infty .
$$

To show this, as above, we prove that

$$
\rho(x)^{\left|\alpha^{\prime}\right|-m-\delta}\left(D^{\beta^{\prime}} \tilde{H}\right)(\Psi(x)) \prod_{j=1}^{\left|\alpha^{\prime}\right|+1-\left|\beta^{\prime}\right|} \zeta_{\beta^{\prime}, j}(x), \quad 2 \leq\left|\beta^{\prime}\right| \leq\left|\alpha^{\prime}\right|,
$$

is bounded. We consider the following cases.

Case 1: $\left|\beta^{\prime}\right| \geq m+1$. Note that (14) equals

$$
\rho(x)^{\left|\beta^{\prime}\right|-m-\delta}\left(D^{\beta^{\prime}} \tilde{H}\right)(\Psi(x)) \rho(x)^{\left|\alpha^{\prime}\right|-\left|\beta^{\prime}\right|} \prod_{j=1}^{\left|\alpha^{\prime}\right|+1-\left|\beta^{\prime}\right|} \zeta_{\beta^{\prime}, j}(x),
$$

which is bounded since

$$
\rho(x)^{\left|\beta^{\prime}\right|-m-\delta}\left(D^{\beta^{\prime}} \tilde{H}\right)(\Psi(x))
$$

is bounded due to the properties of $H$, and

$$
\rho(x)^{\left|\alpha^{\prime}\right|-\left|\beta^{\prime}\right|} \prod_{j=1}^{\left|\alpha^{\prime}\right|+1-\left|\beta^{\prime}\right|} \zeta_{\beta^{\prime}, j}(x)
$$

is bounded due to (ii) and Lemma 2.12 .

Case 2: $\left|\beta^{\prime}\right| \leq m$ and $2 \leq\left|\beta^{\prime}\right| \leq\left|\alpha^{\prime}\right|-m$. By the induction hypothesis, (11), Lemma 2.12, and Remark 2.13,

$$
\rho(x)^{\left|\alpha^{\prime}\right|-m-\delta} \prod_{j=1}^{\left|\alpha^{\prime}\right|+1-\left|\beta^{\prime}\right|} \zeta_{\beta^{\prime}, j}(x)
$$


is bounded. We know that $\left(D^{\beta^{\prime}} \tilde{H}\right)(\Psi(x))$ is bounded as well. Thus (14) is bounded.

Case 3: $\left|\beta^{\prime}\right| \leq m$ and $\left|\alpha^{\prime}\right|-m+1 \leq\left|\beta^{\prime}\right| \leq\left|\alpha^{\prime}\right|$. Note that

$$
\prod_{j=1}^{\left|\alpha^{\prime}\right|+1-\left|\beta^{\prime}\right|} \zeta_{\beta^{\prime}, j}(x)
$$

is bounded due to (11) and the fact that $\left|\alpha^{\prime}\right|-\left|\beta^{\prime}\right|+1 \leq m$. Also note that, for points where $\rho(x) \geq 1$,

$$
\rho(x)^{\left|\alpha^{\prime}\right|-m-\delta}\left|\left(D^{\beta^{\prime}} \tilde{H}\right)(\Psi(x))\right| \leq \rho(x)^{\left|\beta^{\prime}\right|-1}\left|\left(D^{\beta^{\prime}} \tilde{H}\right)(\Psi(x))\right|
$$

because $\left|\beta^{\prime}\right|-1 \geq\left|\alpha^{\prime}\right|-m-\delta$. We know that the right hand side of the above inequality is bounded. For points where $\rho(x)<1$, $\rho(x)^{\left|\alpha^{\prime}\right|-m-\delta}\left(D^{\beta^{\prime}} \tilde{H}\right)(\Psi(x))$ is bounded because $\left|\alpha^{\prime}\right|-m-\delta \geq 0$ and $\left|\beta^{\prime}\right| \leq m$. Thus (14) is bounded.

For $k=1,2, \cdots, 1<p<\infty$, and $-1<r<k p-1$, let $l, m$, and $\delta$ be those in Assumption 2.8 corresponding to $k, p$, and $r$. That is, if $k-\frac{r+1}{p}<1$, then $m=0$ and $\delta=1$. If $k-\frac{r+1}{p} \geq 1$, then $m=\left[k-\frac{r+1}{p}\right]$ and $\delta$ is a number such that $0<\delta<1$ and

$$
k-\frac{r+1}{p}<m+\delta<k-\frac{r+1}{p}+1 .
$$

Assume that we have a function $h \in C^{m, \delta}\left(\mathbb{R}^{d-1}\right), h(0, \cdots, 0)=0$, with compact support, and set $U$ to be an open set

$$
U=\left\{\left(x_{1}, x^{\prime}\right) \in \mathbb{R}^{d}: x_{1}>h\left(x^{\prime}\right)\right\} .
$$

Then we have mappings $\Psi$ and $\Phi$ between $U$ and $\mathbb{R}_{+}^{d}$ enjoying the properties in Lemma 2.14. Using these diffeomorphisms $\Psi$ and $\Phi$, we prove the following lemma.

Lemma 2.15. Let $B_{R}$ be a ball centered at origin with radius $R>0$. For a function $u \in W_{p, r}^{k}(U)$ such that $u(x)=0$ if $x \in \bar{U} \backslash B_{R}$, set $v(y)=u(\Phi(y))$. Then

$$
v \in W_{p, r}^{k}\left(\mathbb{R}_{+}^{d}\right) \quad \text { and } \quad\|v\|_{W_{p, r}^{k}\left(\mathbb{R}_{+}^{d}\right)} \leq c\|u\|_{W_{p, r}^{k}(U)},
$$

where $c$ is independent of $u$. (However, it depends on R.) Similarly, for a function $v \in W_{p, r}^{k}\left(\mathbb{R}_{+}^{d}\right)$ such that $v(y)=0$ if $y \in \overline{\mathbb{R}_{+}^{d}} \backslash B_{R}$, set 
$u(x)=v(\Psi(x))$. Then

$$
u \in W_{p, r}^{k}(U) \quad \text { and } \quad\|u\|_{W_{p, r}^{k}(U)} \leq c\|v\|_{W_{p, r}^{k}\left(\mathbb{R}_{+}^{d}\right)},
$$

where $c$ is independent of $v$. (However, it depends on $R$.)

Proof. We prove the first assertion. Since $\operatorname{det}(\partial \Phi / \partial y)=\partial \Phi_{1} / \partial y_{1}>$ $1 / 2$, it is easy to see that

$$
\|v\|_{L_{p}\left(\mathbb{R}_{+}^{d}\right)} \leq c\|u\|_{L_{p}(U)} .
$$

Now we prove the following inequality to complete the proof of the first assertion.

$$
\sum_{|\alpha|=k} \int_{\mathbb{R}_{+}^{d}}\left|D^{\alpha} v\right|^{p} y_{1}^{r} d y \leq c\|u\|_{W_{p, r}^{k}(U)}^{p} .
$$

Recall that $v(y)=u(\Phi(y))$. Thus by Lemma $2.11^{*}$ the above inequality is proved if we have

$$
\int_{\mathbb{R}_{+}^{d}}\left|D^{\beta} u(\Phi(y)) \prod_{j=1}^{k+1-|\beta|} \zeta_{\beta, j}(y)\right|^{p} y_{1}^{r} d y \leq c\|u\|_{W_{p, r}^{k}(U)}^{p}
$$

for $1 \leq|\beta| \leq k$, where $\zeta_{\beta, j}(y)$ are those defined in Lemma 2.11 with $\Phi$ in place of $\Psi$.

To prove (16), we begin with the case $k-\frac{r+1}{p}<1$. That is, $m=0$ and $\delta=1$. We rewrite the left hand side of (16) as

$$
\int_{\mathbb{R}_{+}^{d}}\left|D^{\beta} u(\Phi(y))\right|^{p} y_{1}^{r-(k-|\beta|) p}\left|y_{1}^{k-|\beta|} \prod_{j=1}^{k+1-|\beta|} \zeta_{\beta, j}(y)\right|^{p} d y .
$$

By Lemma 2.14 and 2.12

$$
y_{1}^{k-|\beta|} \prod_{j=1}^{k+1-|\beta|} \zeta_{\beta, j}(y)
$$

is bounded. Upon recalling (12), we also have

$$
\int_{\mathbb{R}_{+}^{d}}\left|D^{\beta} u(\Phi(y))\right|^{p} y_{1}^{r-(k-|\beta|) p} d y \leq c \int_{U}\left|D^{\beta} u(x)\right|^{p} \rho(x)^{r-(k-|\beta|) p} d x
$$

${ }^{*}$ Lemma 2.11 works for $u$ and $\Psi$ given here. To justify this, one can use almost the same argument as in the proof of Theorem 3.35 in [1] (also use the fact that $\left.u \in W_{p, \text { loc }}^{k}(U)\right)$. Needless to say, $D^{\alpha} v$ and $D^{\beta} u$ in the formula are derivatives in the sense of distributions (or generalized derivatives). 
where the right-hand side is, by Lemma 2.4 (ii) and the fact that $k-\frac{r+1}{p}<$ 1 , less than or equal to a constant times $\|u\|_{W_{p, r}^{k}(U)}^{p}$. Therefore, (16) is proved.

We now prove (16) when $k-\frac{r+1}{p} \geq 1$. In this case, as we recall, we have $m=\left[k-\frac{r+1}{p}\right]$ and $0<\delta<1$, where $\delta$ satisfies (15). We consider two cases depending on whether $\beta$ satisfies $1 \leq k+1-|\beta| \leq m$ or $m<k+1-|\beta|$.

If $1 \leq k+1-|\beta| \leq m$, then

$$
\prod_{j=1}^{k+1-|\beta|} \zeta_{\beta, j}(y)
$$

is bounded. This and (12) imply that the left-hand side of (16) is not greater than a constant times

$$
\int_{U}\left|D^{\beta} u(x)\right|^{p} \rho(x)^{r} d x .
$$

This and Lemma 2.4 (i) (recall that $u$ has compact support) prove (16).

If $m<k+1-|\beta|$, then we rewrite the left hand side of (16) as

$$
\int_{\mathbb{R}_{+}^{d}}\left|D^{\beta} u(\Phi(y))\right|^{p} y_{1}^{r+\delta p-(k-\max \{|\beta|, m\}) p}\left|y_{1}^{k-\max \{|\beta|, m\}-\delta} \prod_{j=1}^{k+1-|\beta|} \zeta_{\beta, j}(y)\right|^{p} d y .
$$

By Lemma 2.14 and 2.12

$$
y_{1}^{k-\max \{|\beta|, m\}-\delta} \prod_{j=1}^{k+1-|\beta|} \zeta_{\beta, j}(y)
$$

is bounded. Upon recalling (12), we also have

$$
\begin{aligned}
& \int_{\mathbb{R}_{+}^{d}}\left|D^{\beta} u(\Phi(y))\right|^{p} y_{1}^{r+\delta p-(k-\max \{|\beta|, m\}) p} d y \\
\leq & c \int_{U}\left|D^{\beta} u(x)\right|^{p} \rho(x)^{r+\delta p-(k-\max \{|\beta|, m\}) p} d x .
\end{aligned}
$$

Note that from (15)

$$
r+\delta p-(k-\max \{|\beta|, m\}) p \geq r-k p+(m+\delta) p>-1 .
$$

Thus by Lemma 2.4 (ii), we have

$$
\int_{U}\left|D^{\beta} u(x)\right|^{p} \rho(x)^{r+\delta p-(k-\max \{|\beta|, m\}) p} d x \leq c\|u\|_{W_{p, r^{\prime}}^{k}(U)}^{p},
$$


where

$$
r^{\prime}=r+\delta p+\max \{m-|\beta|, 0\} p, \quad-1<r^{\prime} \leq k p .
$$

Since $r^{\prime}>r$ and $u$ has compact support, the last term in (17) is not greater than a constant times $\|u\|_{W_{p, r}^{k}(U)}^{p}$. Therefore, (16) is proved. The second assertion follows the same way as the first one. The lemma is proved.

Remark 2.16. Using the above lemma as well as Theorem 3.2 .2 and Remark 3.2.2/2 in [17], one can prove that, for a function $u \in W_{p, r}^{k}(U)$ as in the lemma, there exists a function in $C^{m, \delta}(\bar{U})$ which is arbitrarily close to $u$ in the norm of $W_{p, r}^{k}(U)$. This together with a partition of unity proves that $C^{m, \delta}(\bar{\Omega})$ is dense in $W_{p, r}^{k}(\Omega)$, where $\Omega$ is a domain satisfying Assumption 2.8. In fact, one may have the denseness of $C^{\infty}(\bar{\Omega})$ in $W_{p, r}^{k}(\Omega)$, where $r$ is in an appropriate range, by combining Lemma 2.4 and density results from [9].

In the following lemma, the normal vector to the boundary is extended onto the whole domain, so that the extension has the desired property mentioned in Remark 2.9

Lemma 2.17. Let $m \geq 2,0 \leq \delta<1$, and $h \in C^{m, \delta}\left(\mathbb{R}^{d-1}\right)$ with compact support. Let $N(x)=\left(N_{1}(x), \cdots, N_{d}(x)\right)$ be the unit inner normal vector field defined on $\partial U$, where $U=\left\{\left(x_{1}, x^{\prime}\right) \in \mathbb{R}^{d}: x_{1}>h\left(x^{\prime}\right)\right\}$. Then there exists a vector field $\tilde{N}(x) \in C^{m-1, \delta}(U)$ such that, on $\partial U, \tilde{N}(x)=N(x)$ and

$$
\frac{\partial \tilde{N}_{i}}{\partial \tilde{N}}=\cdots=\frac{\partial^{m-1} \tilde{N}_{i}}{\partial \tilde{N}^{m-1}}=0
$$

for all $i=1, \cdots, d$. Moreover, for $u \in C^{m, \delta}(U)$,

$$
\frac{\partial^{j} u}{\partial \tilde{N}^{j}}=\sum_{|\alpha|=j} D^{\alpha} u \tilde{N}^{\alpha} \quad \text { on } \quad \partial U, \quad j=1, \cdots, m .
$$

Proof. We first see that $N \in C^{m-1, \delta}\left(\mathbb{R}^{d-1}\right.$ ) (a function of $x^{\prime}$ ) and $N_{1}>0$. Define a mapping $\tilde{\Phi}$ from $\overline{\mathbb{R}_{+}^{d}}$ into $\bar{U}$ (one can find this map, e.g. in $[16])$ by

$$
\tilde{\Phi}(y)=\left[\begin{array}{l}
\tilde{\Phi}_{1}(y) \\
\tilde{\Phi}_{2}(y) \\
\cdots \\
\tilde{\Phi}_{d}(y)
\end{array}\right]=\left[\begin{array}{c}
h\left(y^{\prime}\right)+y_{1} N_{1}\left(y^{\prime}\right) \\
y_{2}+y_{1} N_{2}\left(y^{\prime}\right) \\
\cdots \\
y_{d}+y_{1} N_{d}\left(y^{\prime}\right)
\end{array}\right] .
$$

Note that $\tilde{\Phi} \in C^{m-1, \delta}\left(\mathbb{R}_{+}^{d}\right)$. By calculating $\partial \tilde{\Phi} / \partial y$ one can find an $\varepsilon>0$ and $\tilde{\Psi}(x)$ such that $\tilde{\Psi}(x)$ is a mapping from $\left\{\left(x_{1}, x^{\prime}\right) \in \mathbb{R}^{d}: h\left(x^{\prime}\right) \leq x_{1} \leq\right.$ 
$\left.h\left(x^{\prime}\right)+\varepsilon\right\}$ into $\mathbb{R}_{+}^{d}$ and $\tilde{\Phi}^{-1}(x)=\tilde{\Psi}(x)$ on $\left\{\left(x_{1}, x^{\prime}\right) \in \mathbb{R}^{d}: h\left(x^{\prime}\right) \leq x_{1} \leq\right.$ $\left.h\left(x^{\prime}\right)+\varepsilon\right\}$.

Now we define $\tilde{N}(x)$ to be

$$
\tilde{N}(x)=N(\tilde{\Psi}(x))=N\left(\tilde{\Psi}_{2}(x), \cdots, \tilde{\Psi}_{d}(x)\right)
$$

on $\left\{\left(x_{1}, x^{\prime}\right) \in \mathbb{R}^{d}: h\left(x^{\prime}\right) \leq x_{1} \leq h\left(x^{\prime}\right)+\varepsilon\right\}$. We extend $\tilde{N}(x)$ to $\left\{\left(x_{1}, x^{\prime}\right) \in \mathbb{R}^{d}: x_{1} \geq h\left(x^{\prime}\right)\right\}$ so that the extension equals $\tilde{N}(x)$ on $\left\{\left(x_{1}, x^{\prime}\right) \in \mathbb{R}^{d}: h\left(x^{\prime}\right) \leq x_{1} \leq h\left(x^{\prime}\right)+\varepsilon / 2\right\}$ and belongs to $C^{m-1, \delta}(U)$. We denote the extension again by $\tilde{N}(x)$.

Since $\tilde{\Psi}_{i}(x)=x_{i}, i=2, \cdots, d$, for $x \in \partial U$, it follows that

$$
\tilde{N}(x)=N(x) \quad \text { on } \quad \partial U .
$$

To prove (18) notice that, for all $i=1, \cdots, d$,

$$
\frac{\partial^{j} \tilde{N}_{i}}{\partial \tilde{N}^{j}}(x)=\frac{\partial^{j} N_{i}}{\partial y_{1}^{j}}(\tilde{\Psi}(x)) \quad j=1, \cdots, m-1
$$

on $\left\{\left(x_{1}, x^{\prime}\right) \in \mathbb{R}^{d}: h\left(x^{\prime}\right) \leq x_{1} \leq h\left(x^{\prime}\right)+\varepsilon / 2\right\}$. This proves (18) because the right hand side is zero. The last assertion follows from (18). The lemma is proved.

Let $h \in C^{m, \delta}\left(\mathbb{R}^{d-1}\right), m \geq 1$, with compact support and $U=\left\{\left(x_{1}, x^{\prime}\right) \in\right.$ $\left.\mathbb{R}^{d}: x_{1}>h\left(x^{\prime}\right)\right\}$. If $m=1$, we set $\tilde{N}(x)=N\left(h\left(x^{\prime}\right), x_{2}, \cdots, x_{d}\right)$, where $N$ is the unit inner normal vector to $\partial U$. In case $m \geq 2$, let $\tilde{N}$ be the one obtained in the above lemma. Using $\tilde{N}$ as well as diffeomorphisms $\Psi$ and $\Phi$ in Lemma 2.14 , we define a vector field $\mathcal{N}$ on $\overline{\mathbb{R}_{+}^{d}}$ by

$$
\mathcal{N}(y)=\left[\frac{\partial \Psi}{\partial x}(\Phi(y))\right] \tilde{N}(\Phi(y)), \quad \text { i.e., } \quad \mathcal{N}_{i}(y)=\sum_{j=1}^{d} \frac{\partial \Psi_{i}}{\partial x_{j}}(\Phi(y)) \tilde{N}_{j}(\Phi(y))
$$

where $i=1, \cdots, d$. We see that $\mathcal{N} \in C^{m-1, \delta}\left(\mathbb{R}_{+}^{d}\right)$. In addition, from the definition of $\Phi(y)$ and the fact that

$$
\left[\frac{\partial \Phi}{\partial y}(y)\right] \mathcal{N}(y)=\tilde{N}(\Phi(y))
$$

we see that $\mathcal{N}\left(0, y^{\prime}\right)$ is a non-tangential vector field on $\partial \mathbb{R}_{+}^{d}$, i.e., there is an $\varepsilon>0$ such that $\mathcal{N}_{1}\left(0, y^{\prime}\right) \geq \varepsilon$ for all $y^{\prime} \in \mathbb{R}^{d-1}$. We also have

$$
\frac{\partial^{j} u}{\partial \tilde{N}^{j}}(x)=\frac{\partial^{j} v}{\partial \mathcal{N}^{j}}(\Psi(x)) \quad \text { on } \quad \bar{U}, \quad j=1, \cdots, m,
$$


where $u \in C^{m, \delta}(\bar{U})$ and $v(y)=u(\Phi(y))$. In addition, by the property of $\tilde{N}$ we have

$$
\sum_{|\alpha|=j} D^{\alpha} u N^{\alpha}(x)=\sum_{|\alpha|=j} D^{\alpha} u \tilde{N}^{\alpha}(x)=\frac{\partial^{j} v}{\partial \mathcal{N}^{j}}(\Psi(x)) \quad \text { on } \quad \partial U .
$$

Using $\mathcal{N}$ above, we have the following version of the trace and extension theorem for $W_{p, r}^{k}\left(\mathbb{R}_{+}^{d}\right)$ (see Theorem 2.9.2/1 in [17]).

Proposition 2.18. Let $l, m$, and $\delta$ be those in Assumption 2.8 corresponding to $k=1,2, \cdots, 1<p<\infty$, and $-1<r<k p-1$. In case $m \geq 1$, assume that we have $h \in C^{m, \delta}\left(\mathbb{R}^{d-1}\right)$ with compact support, and let $\mathcal{N} \in C^{m-1, \delta}\left(\mathbb{R}_{+}^{d}\right)$ be the vector field described above. Then for $v \in W_{p, r}^{k}\left(\mathbb{R}_{+}^{d}\right)$, we have

$$
\sum_{j=0}^{l-1}\left\|\frac{\partial^{j} v}{\partial \mathcal{N}^{j}}\left(0, y^{\prime}\right)\right\|_{B_{p, p}^{k-\frac{r+1}{p}-j}\left(\mathbb{R}^{d-1}\right)} \leq c\|v\|_{W_{p, r}^{k}\left(\mathbb{R}_{+}^{d}\right)} .
$$

Moreover, for $g_{j} \in B_{p, p}^{k-\frac{r+1}{p}-j}\left(\mathbb{R}^{d-1}\right), \quad j=0, \cdots, l-1$, there exists $v \in W_{p, r}^{k}\left(\mathbb{R}_{+}^{d}\right)$ satisfying

$$
\|v\|_{W_{p, r}^{k}\left(\mathbb{R}_{+}^{d}\right)} \leq c \sum_{j=0}^{l-1}\left\|g_{j}\right\|_{B_{p, p}^{k-\frac{r+1}{p}-j}\left(\mathbb{R}^{d-1}\right)}, \quad \frac{\partial^{j} v}{\partial \mathcal{N}^{j}}\left(0, y^{\prime}\right)=g_{j}\left(y^{\prime}\right) .
$$

This proposition follows from Theorem 2.9.2/1 in [17] (more precisely, Theorem 2.9.2/1 in [17] and the denseness of smooth functions in $\left.W_{p, r}^{k}\left(\mathbb{R}_{+}^{d}\right)\right)$. We here only give the following example to support the proposition. For $k-(r+1) / p>2$, we have

$$
\frac{\partial^{2} v}{\partial \mathcal{N}^{2}}=\sum_{i, j=1}^{d} \frac{\partial^{2} v}{\partial y_{i} \partial y_{j}} \mathcal{N}_{i} \mathcal{N}_{j}+\sum_{i, j=1}^{d} \frac{\partial v}{\partial y_{i}} \frac{\partial \mathcal{N}_{i}}{\partial y_{j}} \mathcal{N}_{j}
$$

Note that

$$
\frac{\partial v}{\partial y_{i}}\left(0, y^{\prime}\right), \quad \frac{\partial^{2} v}{\partial y_{i} \partial y_{j}}\left(0, y^{\prime}\right) \in B_{p, p}^{k-\frac{r+1}{p}-2}\left(\mathbb{R}^{d-1}\right)
$$

and

$$
\mathcal{N}_{j}\left(0, y^{\prime}\right), \quad \frac{\partial \mathcal{N}_{i}}{\partial y_{j}}\left(0, y^{\prime}\right) \in C^{m-2, \delta}\left(\mathbb{R}^{d-1}\right)
$$


Hence, from the fact that $k-(r+1) / p<m+\delta$ and the multiplier theorem (e.g. see [18]), it follows that

$$
\frac{\partial^{2} v}{\partial \mathcal{N}^{2}}(0, y) \in B_{p, p}^{k-(r+1) / p-2}\left(\mathbb{R}^{d-1}\right) .
$$

Remark 2.19. In proving the above proposition, if the vector field $\mathcal{N}$ were $k$-times differentiable, then one could use the argument in Remark 3.6.1/3 in [17], which make use of a diffeomorphism mapping $\mathcal{N}$ to the $y_{1}$ direction. However, depending on $k, p$, and $r$, the vector field $\mathcal{N}$ may not be smooth enough to use the argument.

The following observation is needed in the proof of Theorem 2.10. Let $W_{p, r}^{k}(\Omega)$ satisfy Assumption 2.8. Then we have $\varphi u \in W_{p, r}^{k}(\Omega)$, where $\varphi \in C^{\infty}(\bar{\Omega})$ and $u \in W_{p, r}^{k}(\Omega)$. Especially, $\|\varphi u\|_{W_{p, r}^{k}(\Omega)} \leq c\|u\|_{W_{p, r}^{k}(\Omega)}$, where $c$ is independent of $u$. This can be proved using Lemma 2.4. We also have $\varphi v \in W_{p, r}^{k}\left(\mathbb{R}_{+}^{d}\right)$ and $\|\varphi v\|_{W_{p, r}^{k}\left(\mathbb{R}_{+}^{d}\right)} \leq c\|v\|_{W_{p, r}^{k}\left(\mathbb{R}_{+}^{d}\right)}$ if $\varphi \in C^{\infty}\left(\overline{\mathbb{R}_{+}^{d}}\right)$ and $v \in W_{p, r}^{k}\left(\mathbb{R}_{+}^{d}\right)$ such that $v \equiv 0$ on $\overline{\mathbb{R}_{+}^{d}} \backslash B_{R}$.

The proof of Theorem 2.10 now requires one more preparation - a partition of unity. Assumption 2.8 and Definition 2.3 allow us to have a finite number of balls $B^{\tau}$ and infinitely differentiable functions $\varphi^{\tau}(x), \tau=$ $0,1, \cdots, M$, defined on $\mathbb{R}^{d}$ such that $\operatorname{supp} \varphi^{\tau} \subset B^{\tau}$ and $\sum_{\tau=0}^{M} \varphi^{\tau}(x)=1$ on $\Omega$. We assume that $B^{0} \cap \partial \Omega=\emptyset$ so that $\sum_{\tau=1}^{M} \varphi^{\tau}(x)=1$ on $\partial \Omega$. According to Definition 2.3, for each $\tau, \tau=1, \cdots, M$, after relabeling and re-orienting the coordinate axes if necessary, we have a function $h^{\tau}$ defined on $\mathbb{R}^{d-1}$ such that $\Omega \cap B^{\tau}=U^{\tau} \cap B^{\tau}$, where $U^{\tau}=\left\{\left(x_{1}, x^{\prime}\right): x_{1}>h^{\tau}\left(x^{\prime}\right)\right\}$. Then we construct diffeomorphisms $\Psi^{\tau}$ and $\Phi^{\tau}$ between $U^{\tau}$ and $\mathbb{R}_{+}^{d}$ satisfying Lemma 2.14. Now we additionally assume that, for each $\tau$, $\tau=1, \cdots, M, \rho_{\partial \Omega}(x)=\rho_{\partial U^{\tau}}(x)$ if $x \in \Omega \cap B^{\tau}$. Finally, we construct infinitely differentiable functions $\eta^{\tau}, \tau=1, \cdots, M$, defined on $\mathbb{R}^{d}$ such that $\operatorname{supp} \eta^{\tau} \subset B^{\tau}$ and $\eta^{\tau}=1$ on $D^{\tau}$, where $D^{\tau}$ is a ball satisfying $\operatorname{supp} \varphi^{\tau} \subset \subset D^{\tau} \subset \subset B^{\tau}$.

Proof. [Proof of Theorem 2.10] We consider only the case $k-(r+$ 1) $/ p>1$. (in case $k-(r+1) / p \leq 1$, no normal vector field is considered, so a simple modification of the following argument proves the case). In this case we have $l \geq 2$ and $m \geq 1$.

Clearly, we need only prove (2) and (3). Let $u \in W_{p, r}^{k}(\Omega)$. In fact, by Lemma 2.16 it is enough to have $u \in C^{m, \delta}(\bar{\Omega})$. The inequality (2) follows if we prove

$$
\sum_{j=0}^{l-1}\left\|\sum_{|\alpha|=j} D^{\alpha}\left(\varphi^{\tau} u\right)\left(\Phi^{\tau}(0, \cdot)\right) N^{\alpha}\left(\Phi^{\tau}(0, \cdot)\right)\right\|_{B_{p, p}^{k-\frac{r+1}{p}-j}\left(\mathbb{R}^{d-1}\right)} \leq c\|u\|_{W_{p, r}^{k}(\Omega)},
$$


for $\tau=1, \cdots, M$. Note that if we let $v^{\tau}(y)=\left(\varphi^{\tau} u\right)\left(\Phi^{\tau}(y)\right)$, then by (19)

$$
\frac{\partial^{j} v^{\tau}}{\partial \mathcal{N}^{\tau j}}\left(0, y^{\prime}\right)=\sum_{|\alpha|=j} D^{\alpha}\left(\varphi^{\tau} u\right)\left(\Phi^{\tau}\left(0, y^{\prime}\right)\right) N^{\alpha}\left(\Phi^{\tau}\left(0, y^{\prime}\right)\right), \quad j=1, \cdots, l-1,
$$

where $\mathcal{N}^{\tau}$ is, as described in the argument before Proposition 2.18, the vector field defined on $\overline{\mathbb{R}_{+}^{d}}$ associated with $\Psi^{\tau}$ (or $\Phi^{\tau}$ ). Then by Proposition 2.18 and Lemma 2.15, the above inequality is proved.

To prove (3), set $f_{j}^{\tau}\left(y^{\prime}\right)=\left(\varphi^{\tau} g_{j}\right)\left(\Phi^{\tau}\left(0, y^{\prime}\right)\right)$, where $j=0, \cdots, l-1$ and $\tau=1, \cdots, M$. Then $f_{j}^{\tau}\left(y^{\prime}\right) \in B_{p, p}^{k-\frac{r+1}{p}-j}\left(\mathbb{R}^{d-1}\right)$ and by Proposition 2.18 there are functions $v^{\tau} \in W_{p, r}^{k}\left(\mathbb{R}_{+}^{d}\right), \tau=1, \cdots, M$, such that

$$
\begin{gathered}
\left\|v^{\tau}\right\|_{W_{p, r}^{k}\left(\mathbb{R}_{+}^{d}\right)} \leq c \sum_{j=1}^{l-1}\left\|f_{j}^{\tau}\right\|_{B_{p, p}^{k-(r+1) / p-j}\left(\mathbb{R}^{d-1}\right)}, \\
\frac{\partial^{j} v^{\tau}}{\partial \mathcal{N}^{\tau j}}\left(0, y^{\prime}\right)=f_{j}^{\tau}\left(y^{\prime}\right), \quad j=1, \cdots, l-1 .
\end{gathered}
$$

Without loss of generality we assume that $v^{\tau} \equiv 0$ on $\overline{\mathbb{R}_{+}^{d}} \backslash \Psi^{\tau}\left(\Omega \cap D^{\tau}\right)$. Set $u(x)=\sum_{\tau=1}^{M} \eta^{\tau}(x) v^{\tau}\left(\Psi^{\tau}(x)\right)$. Then the estimate in (3) holds true. Moreover, since $v^{\tau}(y)=0$ if $\eta^{\tau}(\Phi(y)) \neq 1$, it follows from (19) that, for each $j=1, \cdots, l-1$,

$$
\begin{gathered}
\sum_{|\alpha|=j} D^{\alpha} u(x) N^{\alpha}(x)=\sum_{\tau=1}^{M} \eta^{\tau}(x) \sum_{|\alpha|=j} D^{\alpha}\left(v^{\tau}\left(\Psi^{\tau}(x)\right)\right) N^{\alpha}(x) \\
=\sum_{\tau=1}^{M} \eta^{\tau}(x) \frac{\partial^{j} v^{\tau}}{\partial \mathcal{N}^{\tau j}}\left(\Psi^{\tau}(x)\right)=\sum_{\tau=1}^{M} \varphi^{\tau}(x) g_{j}(x)=g_{j}(x)
\end{gathered}
$$

on $\partial \Omega$. This ends the proof.

\section{Traces of Sobolev-Slobodeckij spaces}

Let $\Omega$ be a domain. For $s \geq 0$ and $1 \leq p \leq \infty$, we consider SobolevSlobodeckij spaces $W_{p}^{s}(\Omega)$. As is well-known, if $s$ is a non-negative integer, then $W_{p}^{s}(\Omega)$ is the usual Sobolev space. If $s=k+\lambda$, where $k$ is a nonnegative integer and $0<\lambda<1$, then $W_{p}^{s}(\Omega)$ is the Slobodeckij space, i.e., the space of all $u \in W_{p}^{k}(\Omega)$ satisfying 
$\|u\|_{W_{p}^{s}(\Omega)}=\|u\|_{W_{p}^{k}(\Omega)}+\sum_{|\alpha|=k}\left(\int_{\Omega} \int_{\Omega} \frac{\left|D^{\alpha} u(x)-D^{\alpha} u(y)\right|^{p}}{|x-y|^{d+\lambda p}} d x, d y\right)^{1 / p}<\infty$.

Marschall [11] proved the following.

Theorem 3.1. Let $\Omega$ be a bounded Lipschitz domain. Suppose that $1<p<\infty, s>1 / p$, and $l$ is a positive integer satisfying $l<s-1 / p+1$. In case $s-1 / p \geq 1$ suppose in addition that $\partial \Omega \in C^{m, \delta}, m+\delta>s-1 / p$, where $m$ is a positive integer and $0 \leq \delta<1$. Then the trace operator $T$

$$
T: W_{p}^{s}(\Omega) \rightarrow \prod_{j=0}^{l-1} B_{p, p}^{s-1 / p-j}(\partial \Omega)
$$

is a surjection. In case $s-1 / p$ is not an integer it has a bounded linear right inverse.

In this section, we improve the above theorem by showing that there is a bounded linear right inverse of $T$ even in the case $s-1 / p$ is an integer. We need the following embedding theorem from [12].

Theorem 3.2. Let $\partial \Omega \in C^{1}$. If $0<r<k p$, then

$$
W_{p, r}^{k}(\Omega) \hookrightarrow B_{p, p}^{k-r / p}(\Omega) .
$$

Let us consider $W_{p}^{s}(\Omega)$, where $s-1 / p$ is a positive integer $k$. In this situation the assumption of Theorem 3.1 means that $\partial \Omega \in C^{k, \delta}, 0<\delta<1$. Now consider a weighted Sobolev space $W_{p, r}^{k+1}(\Omega), r=p-1$. Notice that

$$
k+1-\frac{r+1}{p}=k<k+1-\frac{r+1}{p}+1 .
$$

Thus by Theorem 2.10, for a given $\left\{g_{0}, \cdots, g_{k-1}\right\} \in \prod_{j=0}^{k-1} B_{p, p}^{k+1-(r+1) / p-j}(\partial \Omega)$, there is a $u \in W_{p, r}^{k+1}(\Omega)$ such that $T u=\left\{g_{0}, \cdots, g_{k-1}\right\}$. Note that

$$
\begin{gathered}
\prod_{j=0}^{k-1} B_{p, p}^{k+1-(r+1) / p-j}(\partial \Omega)=\prod_{j=0}^{k-1} B_{p, p}^{s-1 / p-j}(\partial \Omega), \\
W_{p, r}^{k+1}(\Omega) \hookrightarrow B_{p, p}^{k+1 / p}(\Omega)=W_{p}^{s}(\Omega),
\end{gathered}
$$

where the last equality holds since $s=k+1 / p$ is not an integer. Therefore, we have proved that, for $\left\{g_{0}, \cdots, g_{k-1}\right\} \in \prod_{j=0}^{k-1} B_{p, p}^{s-1 / p-j}(\partial \Omega)$, there exists $u \in W_{p}^{s}(\Omega)$ such that $T u=\left\{g_{0}, \cdots, g_{k-1}\right\}$, where $k=s-1 / p$. This implies 
that the operator $T$ in Theorem 3.1 has a bounded linear right inverse even if $s-1 / p$ is an integer.

\section{References}

[1] R.A. Adams. Sobolev Spaces, Academic Press, New York-London, 1975.

[2] G.C. Barozzi, Norme equivalenti su certi spazi di Sobolev con peso., Boll. Un. Mat. Ital. (4) (1971), 395-406.

[3] G.M. Constantine and T.H. Savits, A multivariate Fà̀ di Bruno formula with applications, Trans. Amer. Math. Soc., 348(2) (1996), 503-520.

[4] Z. Ding, A proof of the trace theorem of Sobolev spaces on Lipschitz domains, Proc. Amer. Math. Soc., 124(2) (1996), 591-600.

[5] D. Gilbarg and L. Hörmander, Intermediate Schauder estimates, Arch. Rational Mech. Anal., 74(4) (1990), 297-318.

[6] L. Hörmander, The boundary problems of physical geodesy, Arch. Rational Mech. Anal., 62(1) (1976), 1-52.

[7] D. Jerison and C.E. Kenig, The inhomogeneous Dirichlet problem in Lipschitz domains, J. Funct. Anal., 130(1) (1995), 216-219.

[8] K.-H. Kim and N.V. Krylov, On the Sobolev space theory of parabolic and elliptic equations in $C^{1}$ domains, SIAM J. Math. Anal. (electronic), 36(2) (2004), 618-642.

[9] A. Kufner, Weighted Sobolev Spaces, John Wiley \& Sons Inc., New York, 1985.

[10] P.I. Lizorkin, Boundary properties of functions from "weight" classes, Soviet Math. Dokl., 1 (1960), 589-593.

[11] J. Marschall, The trace of Sobolev-Slobodeckij spaces on Lipschitz domains, Manuscripta Math., 58(1-2) (1987), 47-65.

[12] S.M. Nikol'skiū, Approximation of functions of Several Variables and Imbedding Theorems, "Nauka", Moscow, 1977.

[13] S.M. Nikol'skiü, P. I. Lizorkin, and N. V. Miroshin, Weighted function spaces and their pplications to the investigation of boundary value problems for degenerate elliptic equations, Izv. Vyssh. Uchebn. Zaved. Mat., (8) (1988), 4-30.

[14] Y.D. Salmanov, Traces of functions from weighted classes on manifolds and converse embedding theorems, Dokl. Akad. Nauk SSSR, 319(4) (1991), 823-826. 
[15] Y.D. Salmanov, On certain imbedding and extension theorems for a weighted class of functions, Anal. Math., 19(4) (1993), 273-295.

[16] V.V. Shan'kov, The averaging operator with variable radius, and the inverse trace theorem, Sibirsk. Mat. Zh., 26(6) (1985), 141-152.

[17] H. Triebel, Interpolation Theory, Function Spaces, Differential Operators, North-Holland Publishing Co., Amsterdam, 1978.

[18] H. Triebel, Theory of Function Spaces, Birkhäuser Verlag, Basel, 1983.

[19] S.V. Uspenskiu Imbedding theorems for classes with weights, Trudy Mat. Inst. Steklov., 60 (1961), 282-303.

[20] H. Wallin, The trace to the boundary of Sobolev spaces on a snowflake, Manuscripta Math., 73(2) (1991), 117-125.

Department of Mathematics and Statistics

University of Ottawa

585 King Edward Avenue

Ottawa, Ontario K1N 6N5

Canada

(E-mail : kdoyoon@uottawa.ca) 


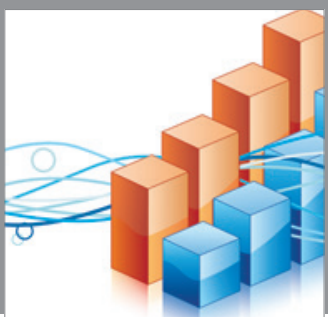

Advances in

Operations Research

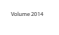

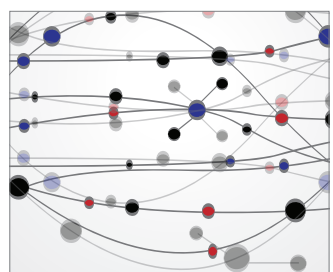

\section{The Scientific} World Journal
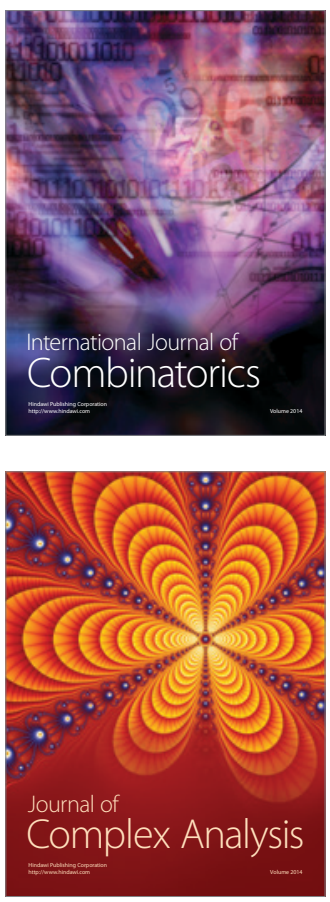

International Journal of

Mathematics and

Mathematical

Sciences
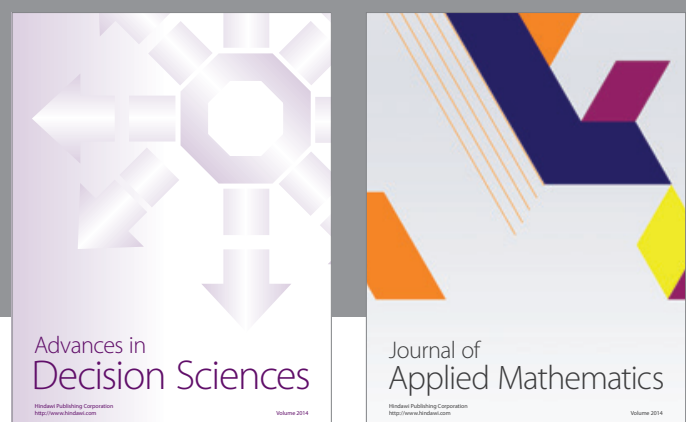

Journal of

Applied Mathematics
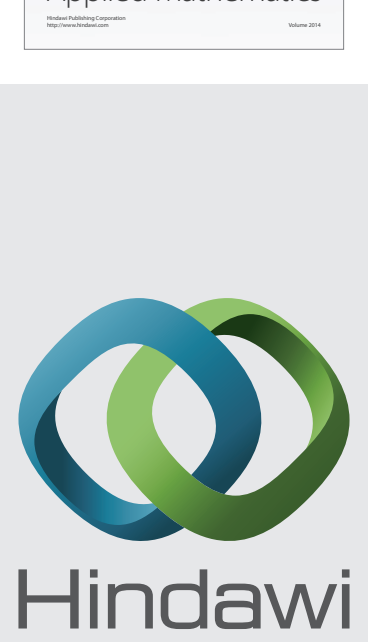

Submit your manuscripts at http://www.hindawi.com
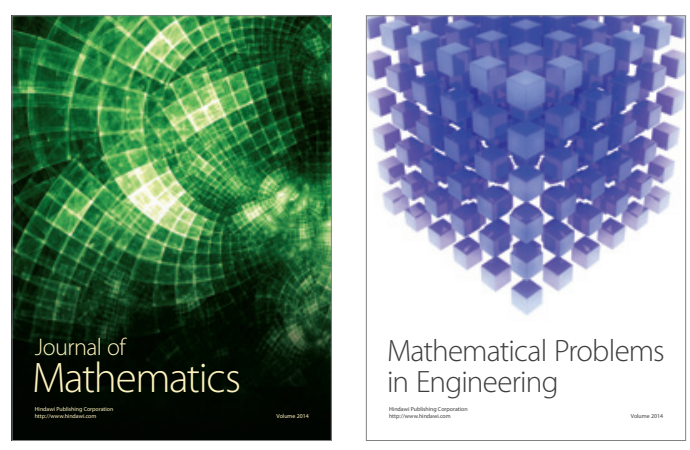

Mathematical Problems in Engineering
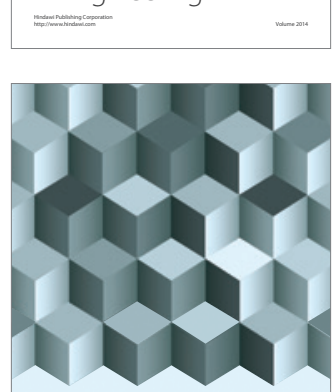

Journal of

Function Spaces
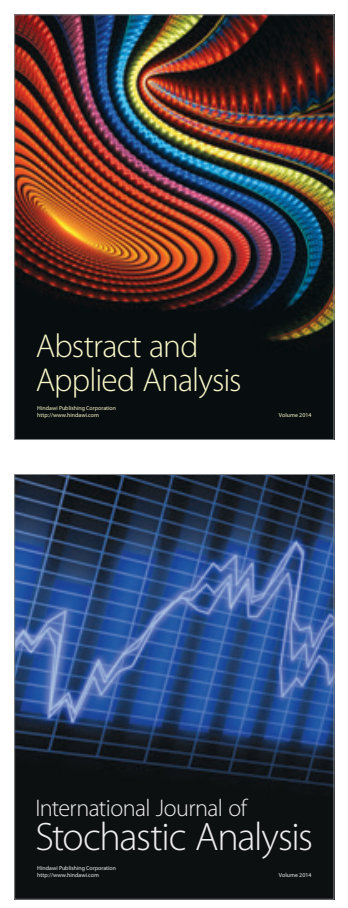

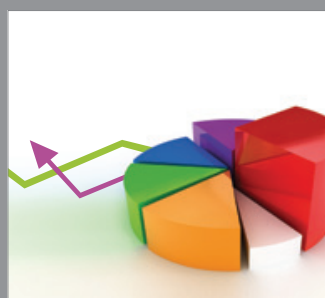

ournal of

Probability and Statistics

Promensencen
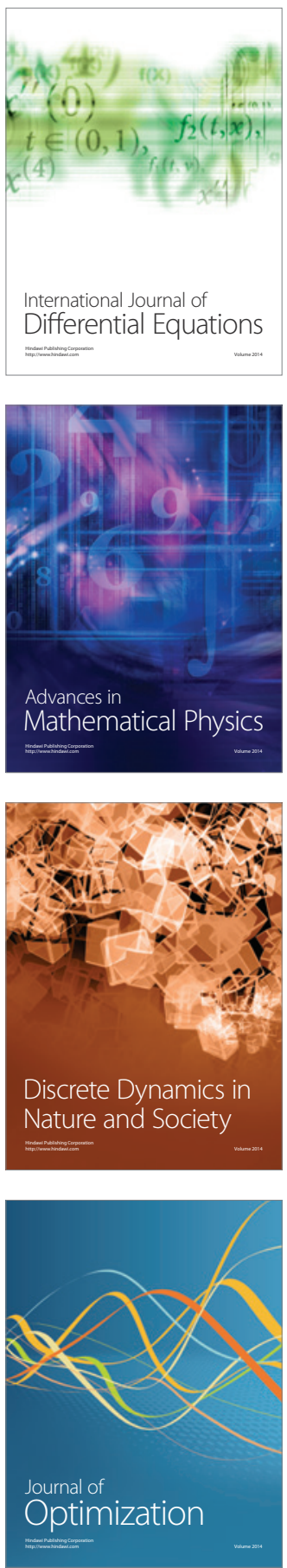